\title{
Salmonella typhimurium and rupture of the thoracic aorta
}

\author{
C B COOPER, J FLINN
}

From the Departments of Respiratory and Communicable Diseases, Lodge Moor Hospital, Sheffield

Transient salmonella bacteraemia is common in patients with gastroenteritis caused by non-typhoidal salmonella ${ }^{1}$ and may result in metastatic infection. Osteomyelitis, arthritis, meningitis, and endocarditis are well recognised. Older patients with atheromatous arterial disease are susceptible to endothelial infection, which may lead to the formation of an aneurysm. ${ }^{2}$ We describe a case of salmonella infection in which the thoracic aorta was infected and which presented with haemoptysis.

\section{Case report}

A 73 year old man sought medical advice because of upper abdominal pain. His symptoms continued despite the prescription of cimetidine. He was referred to hospital and admitted for upper gastrointestinal endoscopy. A duodenal ulcer was seen and he was discharged taking ranitidine. The chest radiograph was normal at this stage.

Within the next two weeks he became feverish and his wife noticed profuse sweating at night. His abdominal pain had improved and there was no diarrhoea. He was readmitted to hospital urgently after developing sudden retrosternal pain and haemoptysis. He described several rigors.

On examination he was febrile $\left(38.5^{\circ} \mathrm{C}\right)$ and hypotensive (blood pressure $100 / 70 \mathrm{~mm} \mathrm{Hg}$ ) and he had tachycardia. His haemoglobin concentration was $10 \cdot 1 \mathrm{~g} / \mathrm{dl}$, white cell count $18.7 \times 10^{6} / 1(89 \%$ neutrophils $)$, platelets $620 \times 10^{9} / 1$, and erythrocyte sedimentation rate $110 \mathrm{~mm}$ in one hour. Serum bilirubin concentration was $32 \mathrm{~g} / 1$, albumin $32 \mathrm{~g} / 1$, alanine transferase $51 \mathrm{IU} / 1$, and $\gamma$ glutamyl transferase $302 \mathrm{IU} / \mathrm{l}$. Chest radiographs now showed an enlarging mass at the left hilum (fig 1). A radionuclide lung scan showed matched defects of ventilation and perfusion. Salmonella typhimurium grew in blood culture and he was transferred to the department of communicable diseases. He was treated with intravenous chloramphenicol $0.5 \mathrm{~g}$ four times daily and, although the pyrexia continued, his condition remained stable. An abdominal ultrasound examination indicated the presence of gall stones.

Nine days after admission he developed pleurisy on the left side associated with profuse haemoptysis. He became hypotensive again and the radial pulses could not be palpated. $S$ typhimurium grew in another blood culture and was now isolated from stool culture. The appearance of the chest radiograph had showed further enlargement of the hilar mass and a flare extending into the left lower lobe (fig 2). Fibreoptic bronchoscopy was contemplated but later that

Address for reprint requests: Dr CB Cooper, University Department of Medicine, Royal Hallamshire Hospital, Sheffield S10 2JF.

Accepted 9 July 1986 day he coughed up a large piece of friable tissue and the procedure was postponed. Subsequent reports indicated that the specimen consisted of inflammatory cells, necrotic tissue, and clotted blood; culture yielded $S$ typhimurium.

Investigation now showed that the haemoglobin concentration was $9.6 \mathrm{~g} / \mathrm{dl}$, the white cell count $19.5 \times 10^{6} / 1$, and the platelet count $590 \times 10^{9} / 1$. He was treated by intravenous infusion of fluid and three units of blood. Metronidazole was added to his drug treatment.

The next day his condition had improved. He remained hypotensive (blood pressure $95 / 75 \mathrm{~mm} \mathrm{Hg}$ ) but now had palpable peripheral pulses and fair urine flow. Later the same day, during a paroxysm of coughing, his blood pressure became unrecordable and he died suddenly.

Necropsy showed a small, clear pleural effusion on the right side. The left hemithorax contained a fresh blood clot weighing $800 \mathrm{~g}$ and about one litre of liquid blood. The thoracic aorta was grossly diseased with fibrous and calcified atheromatous plaques. There were areas of ulceration with adherent mural thrombi. There was a saccular aneurysm of the descending aorta $5 \mathrm{~cm}$ in diameter, which appeared to have eroded the left lower lobe and was surrounded by compressed lung. It had ruptured through a tear in the media about $7 \mathrm{~cm}$ below the origin of the left subclavian artery and communicated with the left lower lobe bronchus. The whole lobe was intensely congested and there was blood within the

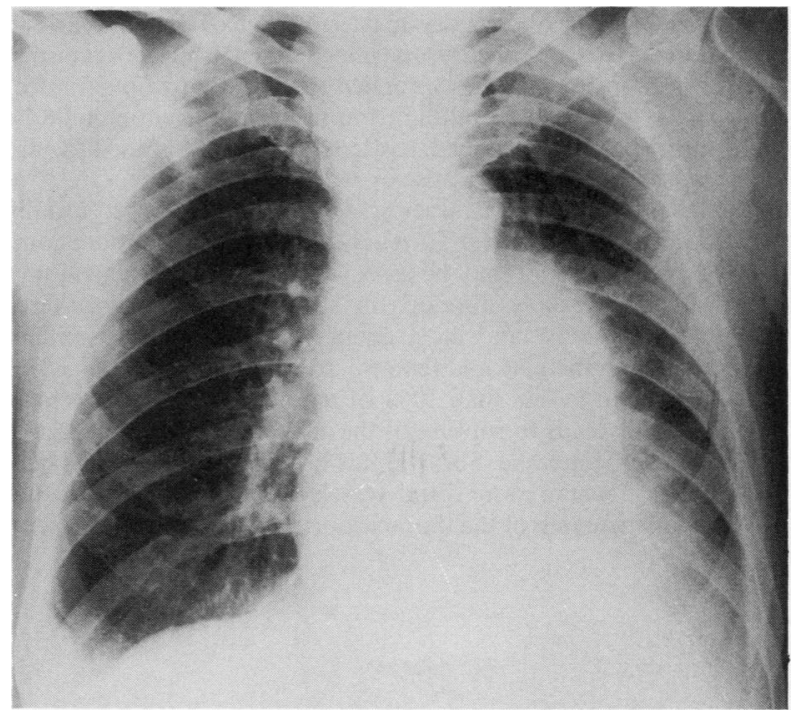

Fig 1 Chest radiograph on admission showing enlarging mass at the left hilum. 


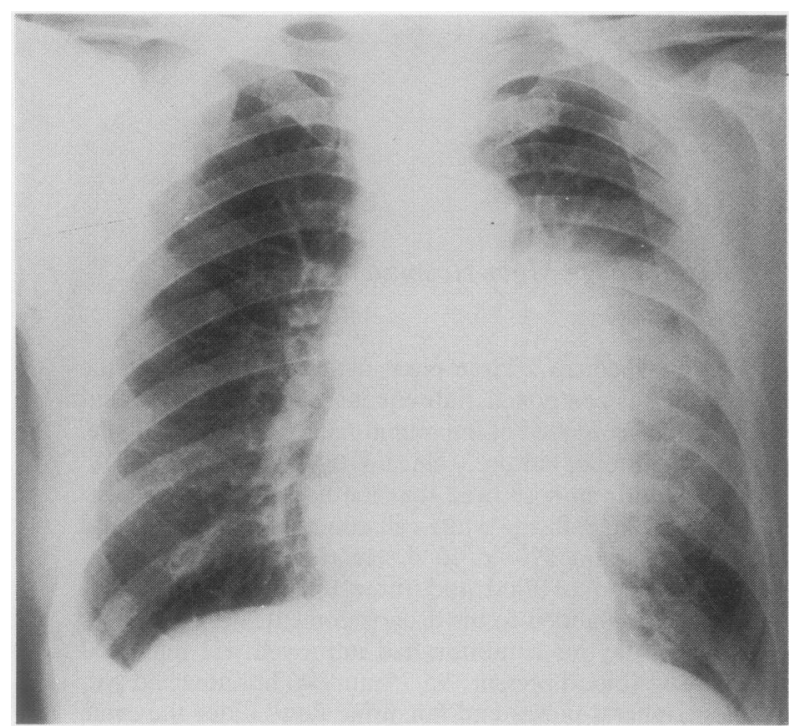

Fig 2 Chest radiograph nine days after admission showing further enlargement of the hilar mass and a flare extending into the left lower lobe.

bronchi. The coronary arteries were extensively affected by atheroma, but there was no evidence of myocardial infarction. The gall bladder was slightly dilated and contained several gall stones. Histological examination of the diseased aortic wall showed a heavy infiltrate of acute inflammatory cells and appearances, which suggested the presence of Gram negative bacilli.

\section{Discussion}

The term mycotic aneurysm was introduced by Osler in 1885 to refer to all aneurysms infected with microorganisms. Since "mycotic" is now applied specifically to fungal infections, aneurysms resulting from bacterial infection could be more accurately named septic aneurysms. About $20 \%$ are caused by salmonella species. ${ }^{3}$

Septic aneurysms due to salmonella infection usually occur in patients over 50 years of age. ${ }^{2}$ They are more common in men and may be associated with diabetes mellitus. ${ }^{4}$ Infection usually develops in arteries affected by atheromatous disease and most aneurysms affect the abdominal aorta and the iliac and femoral arteries. ${ }^{5}$ The thoracic aorta is affected in less than $10 \%$ of reported cases. ${ }^{6}$ Progressive infection leads to rupture of the affected vessels and death is the usual outcome. Survival has been reported after surgical excision where more distal vessels are affected. ${ }^{6-8}$ Rupture of an aneurysm of the thoracic aorta is commonly associated with pain, but haemoptysis is a recognised complication When it occurs it is usually due to direct erosion of the lung by the aneurysm.

Endothelial infection should be suspected when there continuing pyrexia and persistent salmonella bacteraemif Pain in the back, chest, or abdomen may suggest the deve类 opment of an aortic aneurysm. Erosion of the lung should suspected with the radiographic changes we describe. Confirmation of the diagnosis requires ultrasound exarte ination, computed tomography, or angiography. Early diag. nosis is important and should lead immediately to surgers. With septic aneurysms of the thoracic aorta the risks of operation are considerable, but without surgery death inev tably results from uncontrolled lung haemorrhage. Concu仿 rent parenteral antibiotic treatment is essential and shoufd continue for at least four weeks. Endothelial infection hats been reported in $25 \%$ of patients over the age of 50 with salmonella bacteraemia. ${ }^{2}$ Salmonella choleraesuis and $\mathscr{B}$ typhimurium together account for more than $60 \%$ of cases, and patients with diabetes mellitus or impaired immunis are particularly susceptible. A life threatening condition may be prevented, in these selected cases, by early treatment of salmonella infection with appropriate antibiotics.

We thank Dr M W McKendrick for allowing us to repose this case and Dr A Kennedy, who performed the postmortem examination.

\section{References}

1 Saphra I, Winter JW. Clinical manifestations of salmonellosis man. An evaluation of 7779 human infections identified at the New York Salmonella Centre. $N$ Engl J Med 1957;256; 1128-34.

2 Cohen PS, O'Brien TF, Schoenbaum SC, Medeiros AA. The ris of endothelial infection in adults with salmonella bacteraemia. Ann Intern Med 1978;89:931-2.

3 Scheld WM, Sande MA. Cardiovascular infections. 51-Endicarditis and intravascular infections. In: Mandell GL, Douglas RG, Bennett JE, eds. Principles and practice of infectioß disease. New York: John Wiley and Sons, 1985:504-30.

4 Black PH, Kunz LJ, Schwarz MN. Salmonellosis-a review of some unusual aspects. $N$ Engl J Med 1960;262:811-7.

5 Tillotson JR, Lerner AM. Mycotic aneurism and endocarditis. Two uncommon complications of salmonella infection in the same patient. Am J Cardiol 1966;18:267-74.

6 Wilson SE, Gordon HE, van Wegenen PB. Salmonella arteritio Arch Surg 1978;113:1163-6.

7 Meade RH, Moran JM. Salmonella arteritis-preoperative diag nosis and cure of Salmonella typhimurium aortic aneuris N Engl J Med 1969;281:310-2.

8 Reichle FA, Tyson RR, Soloff LA, Lautsch EV, Rosemond GP? Salmonellosis and aneurism of the distal abdominal aorta. Amo Surg 1970;171:219-28.

9 Barbash GI, Solomon A, Reider-Grosswasser E, Vidne BAJ Almog C. Aortic dissection presenting with haemoptysis: diagnostic confirmation of dissection and leak by computed tomography. Heart Lung 1983;12:633-5. 